\title{
Quantum cascade laser light propagation through hollow silica waveguides
}

\author{
D. Francis $\cdot$ J. Hodgkinson $\cdot$ B. Livingstone $\cdot$ \\ R. P. Tatam
}

Received: 31 October 2014 / Accepted: 20 February 2015 / Published online: 1 April 2015

(c) The Author(s) 2015. This article is published with open access at Springerlink.com

\begin{abstract}
In this paper, the transmission characteristics of hollow silica waveguides with bore diameters of 300 and $1000 \mu \mathrm{m}$ are investigated using a $7.8-\mu \mathrm{m}$ quantum cascade laser system. We show that the bore diameter, coiling and launch conditions have an impact on the number of supported modes in the waveguide. Experimental verification of theoretical predictions is achieved using a thermal imaging camera to monitor output intensity distributions from waveguides under a range of conditions. The thermal imaging camera allowed for more detailed images than could be obtained with a conventionally used beam profiler. The results show that quasi-single-mode transmission is achievable under certain conditions although guided single-mode transmission in coiled waveguides requires a smaller bore diameter-to-wavelength ratio than is currently available. Assessment of mode population is made by investigating the spatial frequency content of images recorded at the waveguide output using Fourier transform techniques.
\end{abstract}

Electronic supplementary material The online version of this article (doi:10.1007/s00340-015-6065-5) contains supplementary material, which is available to authorized users.

D. Francis $\cdot$ J. Hodgkinson $(\bowtie) \cdot$ R. P. Tatam

Engineering Photonics, School of Engineering, Cranfield

University, Cranfield MK43 0AL, UK

e-mail: j.hodgkinson@ cranfield.ac.uk

B. Livingstone

Cascade Technologies, Glendevon House, Castle Business Park,

Stirling FK9 4TZ, UK

\section{Introduction}

Hollow waveguides [1] were developed in the 1970s for transmitting infrared radiation at a time when researchers were keen to develop alternatives to chalcogenidebased IR fibres, which exhibit high losses and are brittle. The research was driven in part by the need to guide highpower beams from $\mathrm{CO}_{2}$ lasers operating at a wavelength of $10.6 \mu \mathrm{m}$ for high precision cutting applications. Hollow silica waveguides (HSWs) consist of a silica tube with bore diameters ranging from about $250 \mu \mathrm{m}$ [2] up to around $1000 \mu \mathrm{m}$ [3]. They are coated internally with a layer of silver which is then exposed to a halogen, which converts the silver surface to a silver halide [4]. This improves reflectivity by more than an order of magnitude, depending on the wavelength and the thickness of the halide layer [5]. The total thickness of the silver/silver halide coating is usually around $1 \mu \mathrm{m}$, with the halide layer ranging from 20 to $80 \%$ of this. A schematic showing the construction of a typical HSW is shown in Fig. 1 with radial thickness values of waveguides supplied by Polymicro Technologies ${ }^{\mathrm{TM}}$ [6].

Spectral attenuation is dependent on the thickness of the silver halide layer, and generally, this is chosen so that regions of lowest loss correspond to the wavelengths of the most popular mid-IR lasers, the $\mathrm{CO}_{2}$ laser $(10.6-\mu \mathrm{m}$-thick halide layer) and the Er:YAG laser $(2.9-\mu \mathrm{m}$-thin halide layer). Spectral attenuation for waveguides designed to transmit in the region of these two operating wavelengths is shown in Fig. 2.

The spectral correspondence of HSW with the region of the electromagnetic spectrum associated with the strongest absorptions of many atmospheric gas species, known as the 'molecular fingerprint region', means that there has been significant interest in them for use as spectroscopic gas cells [7]. This has increased rapidly in recent years since 


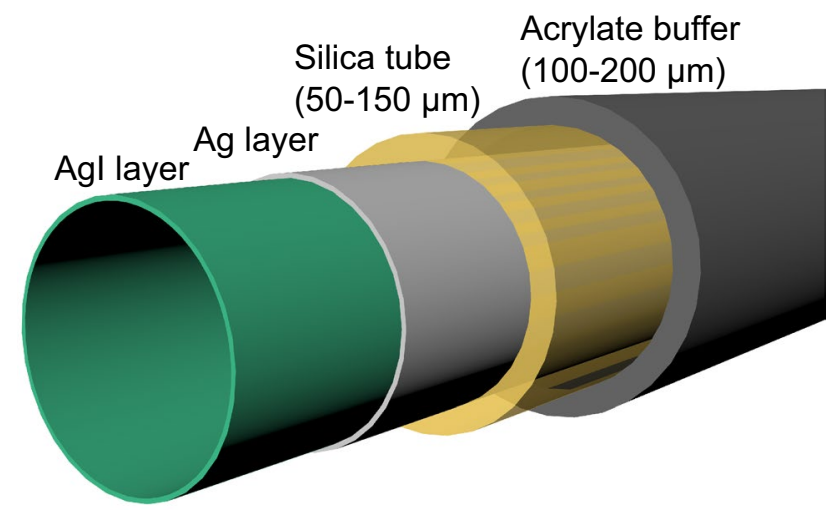

Fig. 1 Different layers within the structure of a hollow silica waveguide. The radial thicknesses of the silica and acrylate layers of the commercially available waveguides from Polymicro Technologies TM [6] with 300 and $1000 \mu \mathrm{m}$ internal bores are shown. The combined thickness of the silver $(\mathrm{Ag})$ and silver iodide (AgI) layers is about $1 \mu \mathrm{m}$

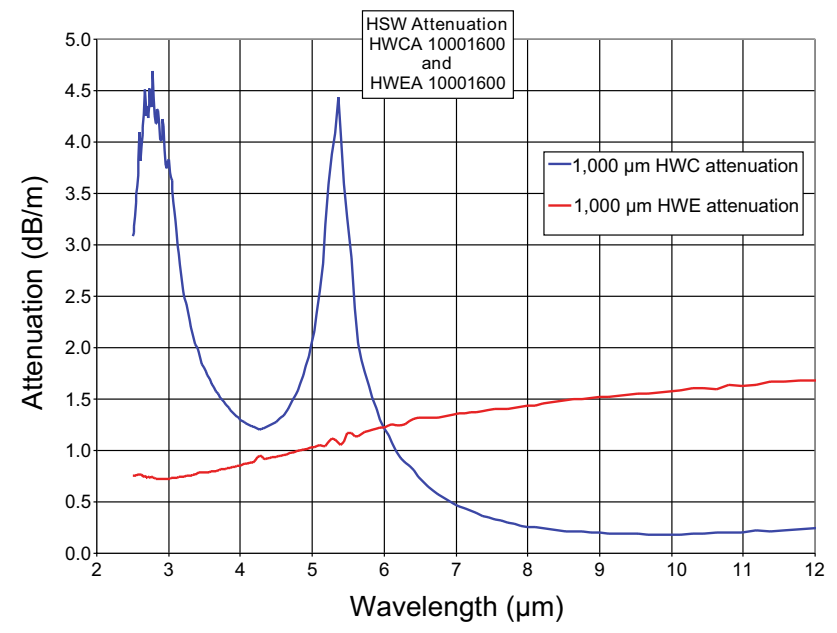

Fig. 2 Spectral attenuation for two different types of hollow silica waveguide available from Polymicro Technologies ${ }^{\mathrm{TM}}$ [6]. Type HWC is designed for use with $\mathrm{CO}_{2}$ lasers $(10.6 \mu \mathrm{m})$, and type HWE is designed for use with Er:YAG lasers $(2.9 \mu \mathrm{m})$. In this work, we used HWC at $7.8 \mu \mathrm{m}$. Reproduced with permission of Polymicro Technologies, a subsidiary of Molex Inc.

the advent of quantum cascade lasers (QCLs) [8, 9], which also have operating wavelengths within this region [10]. HSW gas cells are particularly advantageous in applications where only small volumes are available and/or where fast response times are required [11].

Transmission properties such as attenuation characteristics, modal transmission and the influence of launch conditions of hollow silica waveguides are of interest. For instance, multimode transmission through a hollow waveguide gas cell may result in temporal dispersion of the signal, causing degradation of the resolved gas absorption features when using very high-bandwidth detectors. These properties have been investigated previously based on the $\mathrm{CO}_{2}$ laser application wavelength [12] at $10.6 \mu \mathrm{m}$, using an established theoretical framework which describes waveguide transmission [13]. The modal properties of a $\mathrm{CO}_{2}$ laser-coupled HSW have also been investigated using a beam profiler to monitor output intensity distributions [14]. Output intensity distributions have also been measured using a beam profiler from continuous-wave QCLcoupled hollow waveguides with wavelengths of 5.27 and $10.5 \mu \mathrm{m}$ [15]. Recently [16], this group has further demonstrated single-mode transmission in $200-\mu \mathrm{m}$-bore-diameter HSW with a series of QCLs with wavelengths ranging from 5.1 to $10.5 \mu \mathrm{m}$. Single-mode transmission was verified by monitoring the output intensity distributions with a beam profiler. Optical transmission through HSW has also been investigated at much shorter wavelengths. Chen et al. [17] observed highly multimode transmission through $750-\mu \mathrm{m}$-bore-diameter waveguides coupled to verticalcavity surface emitting lasers (VCSELs) with wavelengths of 1.6 and $2.3 \mu \mathrm{m}$. The multimode transmission produced speckle noise caused by interference between different modes. This led to a reduction in spectral absorbance resolution; however, this was improved by an order of magnitude by mechanically vibrating the waveguide.

Here we are interested in the transmission properties of a pulsed QCL operating at $7.8 \mu \mathrm{m}$ which is used to make spectroscopic measurements of methane concentrations via the intra-pulse technique [18]. In Sect. 2, transmission properties of $7.8 \mu \mathrm{m}$ radiation through waveguides with the same dimensions as those available from Polymicro Technologies ${ }^{\mathrm{TM}}$ are investigated using established theory in a manner similar to that presented by Nubling and Harrington [12]. The intra-pulse spectroscopic technique is discussed in Sect. 3. The findings of the investigation presented in Sect. 2 are verified experimentally using a thermal imaging camera to image output intensity distributions, and these results are given in Sect. 4. The images obtained with the thermal imaging camera results are compared with those obtained with a micro-bolometer array, and better resolution is shown to be obtained with the former. Here we quantify the modal properties using the mean spatial frequency of the resulting images.

\section{Electromagnetic mode propagation in hollow silica waveguides}

From a classical viewpoint, a mode within a waveguide represents one of the possible paths a ray of light can take through it. For instance, solid-core single-mode silica optical fibres allow for rays to follow only one path; however, as the diameter of the core increases, the number of supported modes also increases. The number of possible 
modes is finite, however, as light is only guided on a discrete set of paths. This is a consequence of the wave nature of light and can be established through analysis of Maxwell's equations [5]. These modes are defined with respect to the polarization of the light wave as transverse electric $\left(\mathrm{TE}_{l m}\right)$, where the electric field is perpendicular to the propagation direction, and transverse magnetic $\left(\mathrm{TM}_{l m}\right)$, where the electric field is parallel to the propagation direction. The two subscript indices are required due to the cylindrical geometry of the fibre and represent the radial $(l)$ and azimuthal $(m)$ dependence of the electric field. In addition, there exist hybrid modes $\mathrm{HE}_{l m}$ and $\mathrm{EH}_{l m}$, where there is a component of the electric field in the propagation direction. These represent skew rays, which do not intersect the optical axis and travel helically through the fibre.

An important parameter, which determines the number of supported modes in an optical fibre, is the $V$-number, which is also known as the normalized frequency and is given by [19]

$V=\frac{2 \pi a \cdot \mathrm{NA}}{\lambda}$

where $a$ is the core radius, $\lambda$ is the optical wavelength, and NA is the numerical aperture of the fibre, which is related to the acceptance angle $\theta_{a}$ by [20]

$\mathrm{NA}=\sqrt{n_{1}^{2}-n_{2}^{2}}=\sin \theta_{a} / 2$

where $n_{1}$ and $n_{2}$ are the refractive indices of the core and cladding, respectively. Fibres with $V$-numbers below 2.405 are always single mode, and for multimode fibres, the number of supported modes is given approximately by

$M \approx \frac{4}{\pi^{2}} V^{2}$

The diameter of a single-mode core designed for visible and near IR wavelengths is typically less than $10 \mu \mathrm{m}$, and a multimode core ranges between 50 and $200 \mu \mathrm{m}$. For infrared optical fibres, for instance, the chalcogenide range supplied by IRFlex TM [21], the core diameters are $9 \mu \mathrm{m}$ for single mode and $200 \mu \mathrm{m}$ or $300 \mu \mathrm{m}$ for multimode. In comparison, HSWs have bore diameters ranging from about 300 to $1000 \mu \mathrm{m}$. It might be expected therefore that HSW would support many more modes than a multimode fibre, even though HSW is designed to guide light at midIR wavelengths which can be an order of magnitude longer than visible wavelengths.

One of the issues with supporting a large number of modes is temporal dispersion [20], which can cause degradation of information encoded in the light by way of a temporal intensity variation. In spectroscopy, this information relates to the spectral absorption features that are being measured. If the time delay between the axial ray (fundamental mode) and the ray that follows the longest path (highest order mode) is greater than the sample period of the detector system, then blurring of the signal will occur, which in spectroscopy would appear as a broadening of the spectral features. This will likely only become a problem for very long cell lengths or very fast detectors, however. For instance, assuming a detector period of $1 \mathrm{~ns}$ and a 7.8- $\mu \mathrm{m}$ source coupled into a straight $1000 \mu \mathrm{m}$ diameter waveguide with an $f / 30$ lens $(5 \mathrm{~mm}$ aperture diameter, $150 \mathrm{~mm}$ focal length), the length of the waveguide would need to be $2.16 \mathrm{~km}$ before temporal dispersion became a problem.

Establishing the number of modes propagating through a hollow silica waveguide is an interesting problem and not straightforward. For one thing, the relations given in Eqs. (1)-(3) are well defined for solid-core silica optical fibres which have refractive indices which are close in value, but a hollow waveguide does not have a conventional cladding material. The inner walls are coated with silver which has a high extinction coefficient $\kappa$, and therefore, the complex refractive index $n^{*}=n-i \kappa$ must be considered, which at $7.8 \mu \mathrm{m}$ is $8.48-i 45.0$ [22]. The NA of a hollow waveguide cannot therefore be simply computed using Eq. (2), and instead, an effective numerical aperture $\mathrm{NA}_{\text {eff }}$ is used which is inferred from measurements [5]. Taking an NA equal to that of an $f / 16$ coupling lens and assuming a $7.8-\mu \mathrm{m}$ source, a $1000 \mu \mathrm{m}$ hollow waveguide would have, if calculated from Eqs. (1) and (3), a $V$-number of 25.2 and a possible number of modes $M$ of 256. It would therefore seem that hollow-core waveguides are heavily multimode; however, this does not turn out to be the case due to the losses experienced by different modes, with higher-order modes being more heavily attenuated. This is because higher-order modes interact with the coating more often per unit length and lose energy on each interaction.

\subsection{Modal attenuation within hollow silica waveguides}

Losses within hollow cylindrical waveguides are dependent on the bore radius $a$ and the bend radius $R$, as given by [5]

$\alpha \approx \frac{1}{a^{3}}$

and

$\alpha \approx \frac{1}{R}$

Typical loss figures for the different commercially available HSW types are given in Table 1 . The $1 / a^{3}$ dependence on loss explains the fourfold increase in the $\mathrm{dB} / \mathrm{m}$ loss when going from $1000 \mu \mathrm{m}$ to $300 \mu \mathrm{m}$ bore diameter. It may appear therefore that to minimize loss, the largest possible bore size is desirable. However, it becomes 
Table 1 Summary of losses associated with HSW available from Polymicro Technologies ${ }^{\mathrm{TM}}[6]$

\begin{tabular}{lll}
\hline Bore diameter $(\mu \mathrm{m})$ & Straight loss $(\mathrm{dB} / \mathrm{m}$ max $)$ & $\begin{array}{l}\text { Bend loss }(\mathrm{dB} \text { max }) \\
\left(360^{\circ} \text { in } 40 \mathrm{~cm} \text { loop }\right)\end{array}$ \\
\hline 300 & 2.0 & 1.5 \\
500 & 0.8 & 1.5 \\
750 & 0.5 & 1.0 \\
1000 & 0.5 & 1.0
\end{tabular}

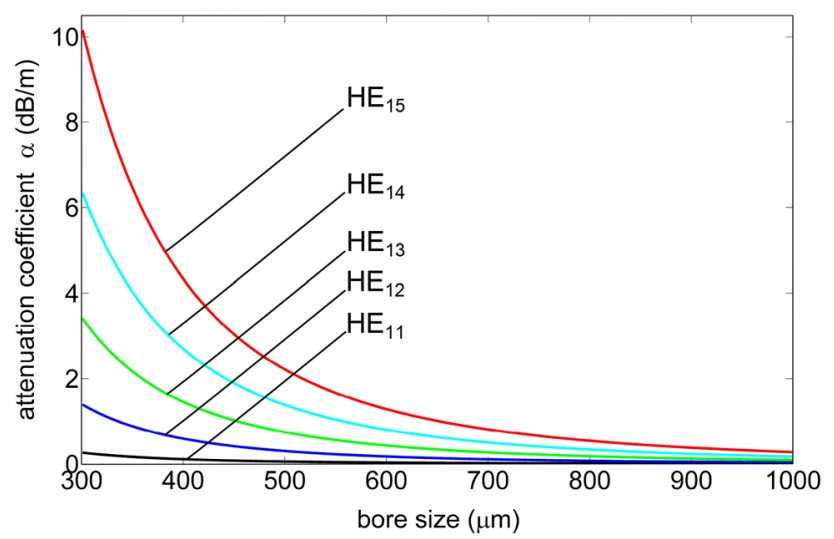

Fig. 3 Variation of the attenuation coefficient $\alpha_{1, \mathrm{~m}}$ with bore diameter for the five lowest order $\mathrm{HE}_{1 \mathrm{~m}}$ modes at $7.8 \mu \mathrm{m}$. The high attenuation of higher-order modes for the smaller bore diameters means that HSW can transmit a single mode under certain conditions if the bore diameter is small enough

impractical to construct hollow silica waveguides with bore diameters much greater than $1000 \mu \mathrm{m}$ due to the inherent reduction in flexibility. Reduced loss can be obtained using hollow polycarbonate waveguides, which can be made with bore diameters up to $2000 \mu \mathrm{m}$ while retaining a good level of flexibility [3].

The propagation of light through cylindrical hollow metallic waveguides was originally analysed by Marcatili and Schmeltzer [23] and was later expanded upon by Miyagi and Kawakami [13] to include analysis of the influence of the dielectric layer. They derived equations that can be used to calculate losses within hollow waveguides for the different mode types. When a Gaussian beam is coupled on-axis into a hollow waveguide, only the $\mathrm{HE}_{1 m}$ modes are populated. In this case, modal attenuation can be calculated using [5]

$\alpha_{l m}=\left(\frac{u_{l m}}{2 \pi}\right)^{2} \frac{\lambda^{2}}{a^{3}}\left(\frac{n}{n^{2}+\kappa^{2}}\right) \times\left\{\frac{1}{2}\left[1+\frac{n_{d}^{2}}{\sqrt{n_{d}^{2}-1}}\right]^{2}\right\}$

where $n$ and $\kappa$ are refractive index and extinction coefficient of the metallic layer, i.e. silver, and $n_{d}$
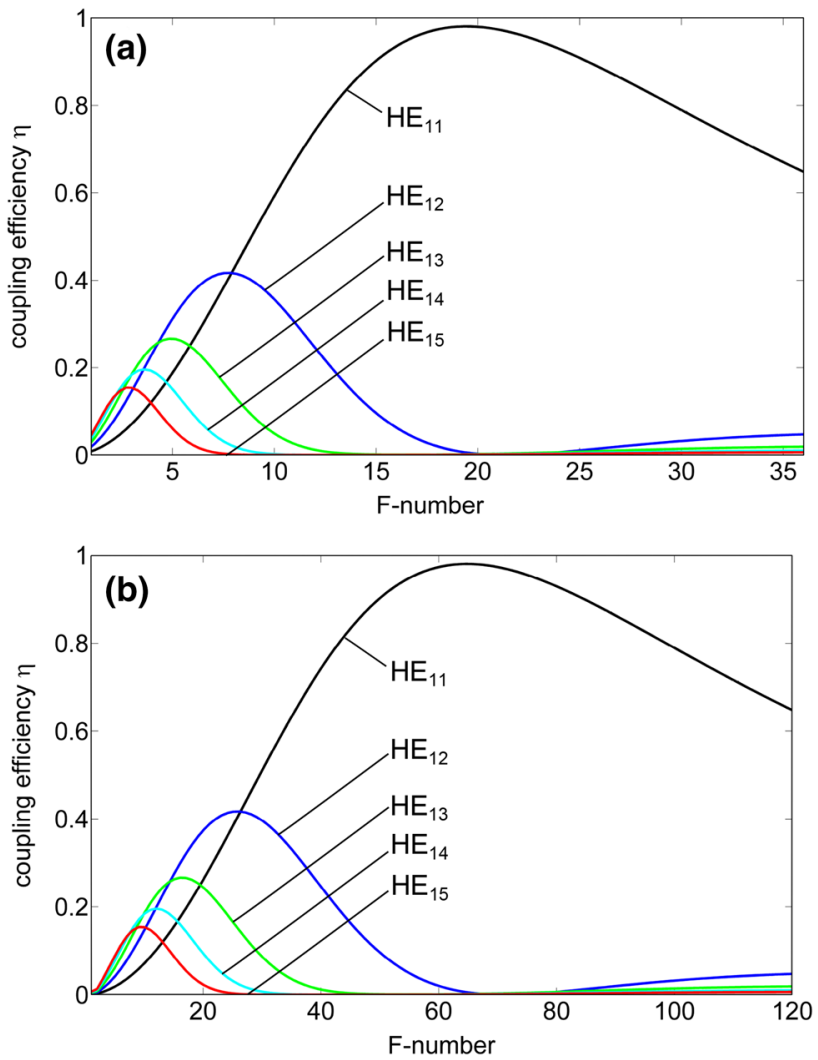

Fig. 4 Coupling efficiency $\eta$ for the five lowest order $\mathrm{HE}_{l m}$ modes plotted against increasing f-number for a a $300 \mu \mathrm{m}$ bore diameter waveguide and $\mathbf{b} 1000 \mu \mathrm{m}$ bore diameter waveguide

is the refractive index of the dielectric layer. The mode parameter $u_{l m}$ is given by the $m$ th zero of the $(l-1)$-order Bessel function. Figure 3 shows the variation of the attenuation coefficient with bore diameter for the first five $\mathrm{HE}_{1 m}$ modes calculated using Eq. (6). These attenuation coefficients were calculated for a wavelength of $7.8 \mu \mathrm{m}$ using the following optical constants: $n=8.48, \kappa=45.0$ and $n_{d}=2.1$ [22]. Ellipsometry can be used to measure these optical constants as demonstrated by George and Harrington [3] who obtained values of $n=4.98, \kappa=33.82$ for silver and $n_{d}=1.95$ for silver iodide at $10.6 \mu \mathrm{m}$. The data show a significant reduction in attenuation for lower-order modes and the $1 / a^{3}$ variation with bore diameter that was highlighted in Eq. (4). This indicates that while waveguides with smaller bore diameters exhibit greater total loss, the attenuation of the higher-order modes is of such an extent that they support much fewer modes. In certain conditions, an HSW can preserve singlemode transmission [2], and it has been stated that this can be achieved when using bore diameters less than about 30 times the operating wavelength, though this also depends on other parameters such as wall 
thickness [14]. On the other hand, while larger bore waveguides may carry a greater number of different modes, they exhibit less loss overall.

\subsection{Influence of launch condition on HSW transmission properties}

The proportion of the light energy entering a waveguide is referred to as the coupling efficiency. The value of the coupling coefficient is dependent on the spatial distribution of the light field in the image plane of the coupling lens and the mode profile of the waveguide. This can be computed from the overlap integral and has been done previously for single-mode fibres [24], and for hollow waveguides [12, $25,26]$,

$\eta_{l m}=\frac{\left[\int_{0}^{a} E_{\text {beam }} E_{\text {waveguide }} r \mathrm{~d} r\right]^{2}}{\int_{0}^{\infty} E_{\text {beam }}^{2} r \mathrm{~d} r \int_{0}^{a} E_{\text {waveguide }}^{2} r \mathrm{~d} r}$

where $E_{\text {beam }}$ is the Gaussian spatial laser beam profile and $E_{\text {waveguide }}$ is the spatial profile of the $\mathrm{HE}_{1 m}$ modes of the waveguide, given by

$E_{\text {beam }}(r)=E_{0} \exp \left(\frac{-r^{2}}{\omega^{2}}\right) \quad E_{\text {waveguide }}(r)=E_{0} J_{0}\left(u_{l m} \frac{r}{a}\right)$

where $0<r<a$ and $J_{0}$ represents the zero-order Bessel function of the first kind. The focused beam waist $\omega$ is related to the $f$-number of the coupling lens by

$f_{\#}=\frac{\pi \omega}{2 \lambda}$

The $f$-number is the ratio of the lens focal length to the aperture diameter and is related to the lens numerical aperture by $f_{\#} \approx 1 / 2 \mathrm{NA}$. Figure 4 a shows how the coupling efficiency varies with $f$-number for a $300 \mu \mathrm{m}$ diameter bore waveguide. The waveguide is assumed to be straight, $1 \mathrm{~m}$ long and guiding a source wavelength of $7.8 \mu \mathrm{m}$. The data show that at an $f$-number of approximately $f / 19$, most of the incident light is coupled into the lowest order $\mathrm{HE}_{11}$ mode. This differs from the $f / 16$ result obtained in Nubling and Harrington's paper [12] for a $320-\mu \mathrm{m}$-bore-diameter waveguide due to the shorter source wavelength used here. Figure $4 \mathrm{~b}$ shows the coupling efficiency variation versus $f$-number for a $1000 \mu \mathrm{m}$ bore diameter waveguide, and in this case, the $f$-number for optimum coupling efficiency into the $\mathrm{HE}_{11}$ mode is approximately $f / 64$.

If single-mode operation is not specifically required, it may be more beneficial to select launch conditions that yield the lowest total loss. The condition for lowest total loss can be achieved by launching with a slightly lower $f$-number lens, producing a smaller beam waist and

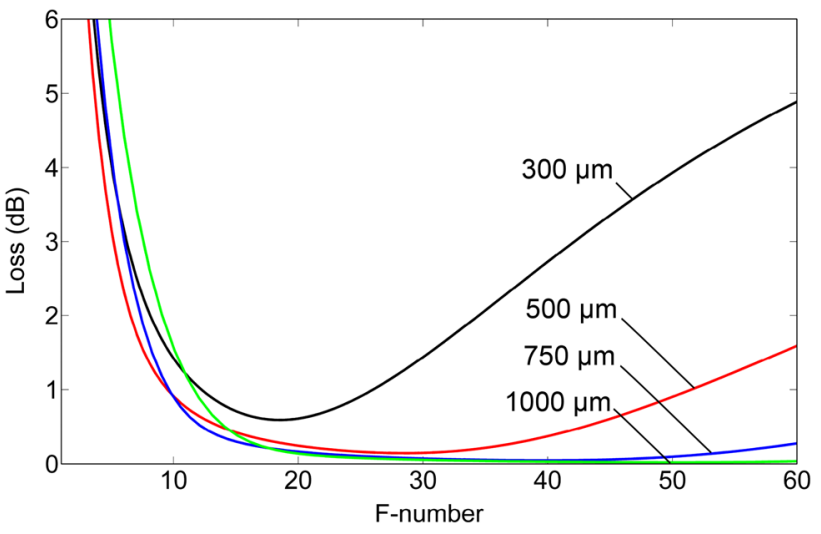

Fig. 5 Calculated loss vs. $f$-number for four different bore diameters

Table 2 Optimum launch conditions for coupling to lowest order mode and for lowest total loss for four different bore sizes based on calculations using Eqs. (9) and (11), respectively

\begin{tabular}{lll}
\hline Bore size $(\mu \mathrm{m})$ & $\begin{array}{l}\text { F-number (optimum } \\
\text { coupling to } \mathrm{HE}_{11} \text { mode) }\end{array}$ & $\begin{array}{l}\text { F-number (lowest total } \\
\text { loss) }\end{array}$ \\
\hline 300 & $\mathrm{f} / 19$ & $\mathrm{f} / 18$ \\
500 & $\mathrm{f} / 32$ & $\mathrm{f} / 29$ \\
750 & $\mathrm{f} / 48$ & $\mathrm{f} / 40$ \\
1000 & $\mathrm{f} / 64$ & $\mathrm{f} / 50$ \\
\hline
\end{tabular}

coupling slightly more energy into the higher-order modes. The transmitted intensity per unit length $z$ can be calculated using [12].

$I(z)=\sum_{m} \eta_{m} \exp \left(-2 \alpha_{m} z\right)$

where $\eta_{m}$ and $\alpha_{m}$ are the coupling efficiencies and attenuation coefficients for the first $m \mathrm{HE}_{1 m}$ modes, respectively. The loss over a 1-m length waveguide plotted against $f$-number of the coupling lens is shown in Fig. 5 for a range of different bore diameters. The first five $\mathrm{HE}_{1 m}$ modes were included in the summation. The optimum $f$-numbers for both conditions for $1 \mathrm{~m}$ lengths of the four different bore diameters are summarized in Table 2. Recently, the variation of loss with launch condition was measured experimentally for a range of QCL wavelengths and showed good agreement with theory [16].

\section{Intra-pulse spectroscopy with a quantum cascade laser}

The laser used in this work was a 7.8- $\mu \mathrm{m}$ QCL housed in the Cascade Technologies ${ }^{\mathrm{TM}}$ CT3000 gas analysis platform [27]. The system can incorporate up to four lasers for 
multispecies analysis; however, for this work, a single laser, which is used for measuring methane, was all that was required. A mercury cadmium telluride $(\mathrm{HgCd}) \mathrm{Te}$ detector with a detectivity of $2.6 \times 10^{9} \mathrm{cmHz}^{1 / 2} / \mathrm{W}$ was used in conjunction with the laser system. Gas concentration measurements were made using the intra-pulse technique [18]. This is a form of tunable laser spectroscopy [28] whereby the wavelength tuning occurs across the laser pulse due to the thermal effect of the pulsing. In contrast, the inter-pulse technique employs frequency tuning via modulation of the control current [29]. For this laser, the chirp rate across the pulse is $0.003 \mathrm{~cm}^{-1} / \mathrm{ns}$, which means that for a $500 \mathrm{~ns}$ pulse
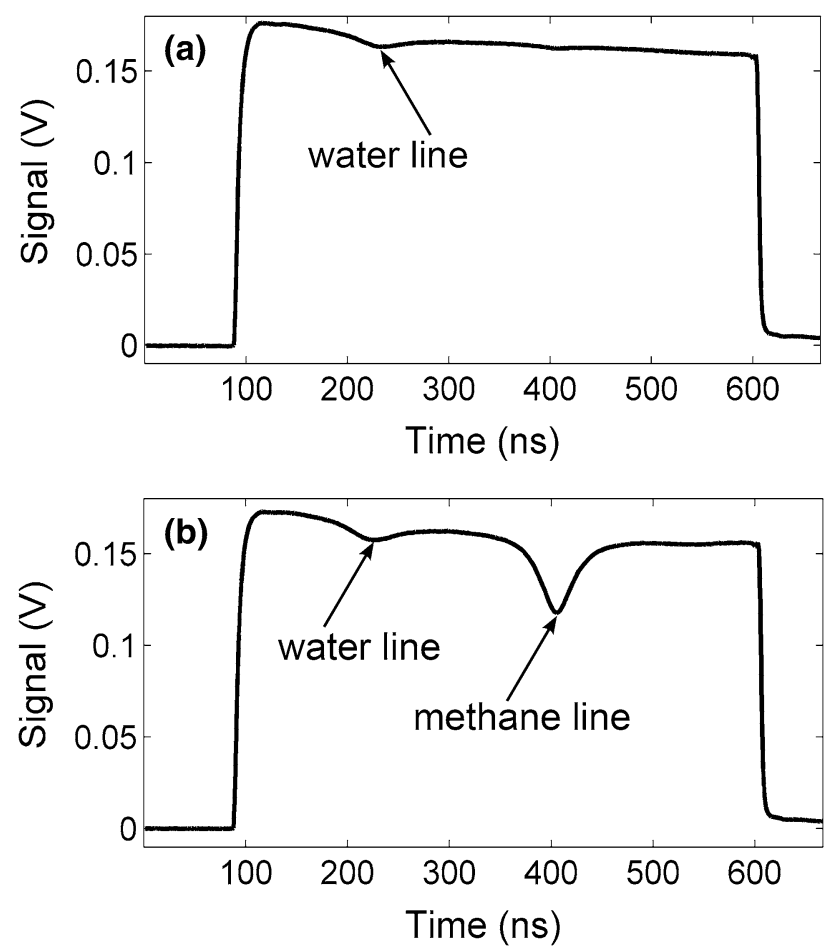

Fig. 6 Intra-pulse QCL spectroscopy: Typical pulses recorded in a laboratory air and $\mathbf{b}$ with the beam passing through $2.5 \%$ methane in a 165-mm-pathlength gas cell emitted at a control temperature of $25{ }^{\circ} \mathrm{C}$, the total wavelength variation across the pulse is $9.2 \mathrm{~nm}$. The temperature tuning rate is $0.09 \mathrm{~cm}^{-1} /{ }^{\circ} \mathrm{C}$, which results in a total wavelength range of $27.5 \mathrm{~nm}$ for a control temperature range of $50{ }^{\circ} \mathrm{C}$.

An example of the spectroscopic principle is shown in Fig. 6. Figure 6a shows a typical digitized recording which consists of an average of 1000 individual pulse measurements captured at a pulse acquisition rate of $50 \mathrm{kHz}$. It was obtained with light from the QCL directly incident on the detector. A small water line can be seen at approximately $230 \mathrm{~ns}$ due to the presence of humidity in the laboratory air. Figure $6 \mathrm{~b}$ is obtained with the QCL beam first passing through a $165-\mathrm{mm}$ gas cell containing methane with a concentration of $2.5 \%$ by volume. An absorption feature is now observable at approximately $410 \mathrm{~ns}$ in the pulse. An advantage of this spectroscopic technique is that the spectral resolution is not dependent on the spectral linewidth of the laser (unlike in the inter-pulse technique), and therefore, a very high spectral resolution can be achieved. Instead, the spectral resolution is dependent on the temporal resolution of the detection system. The data shown in Fig. 6 have a temporal resolution of $1.33 \mathrm{~ns}$. Such high detection rates come at the cost of limited digital resolution; however, digitization errors can be minimized with averaging.

\section{Investigation of HSW modal properties with a thermal imaging camera}

In order to experimentally verify the observations made in Sect. 3, a thermal imaging camera (FLIR Systems Thermacam SC3000) was used to image output intensity distributions from hollow silica waveguides. We investigated HSW with bore diameters of 300 and $1000 \mu \mathrm{m}$ because they represent the minimum and maximum of those available commercially. The camera sensor consists of an array of gallium arsenide (GaAs) quantum well infrared photodetectors $(\mathrm{QWIP})$ with $320 \times 240$ pixels. The spectral response peaks over the range $8-9 \mu \mathrm{m}$ and drops to about

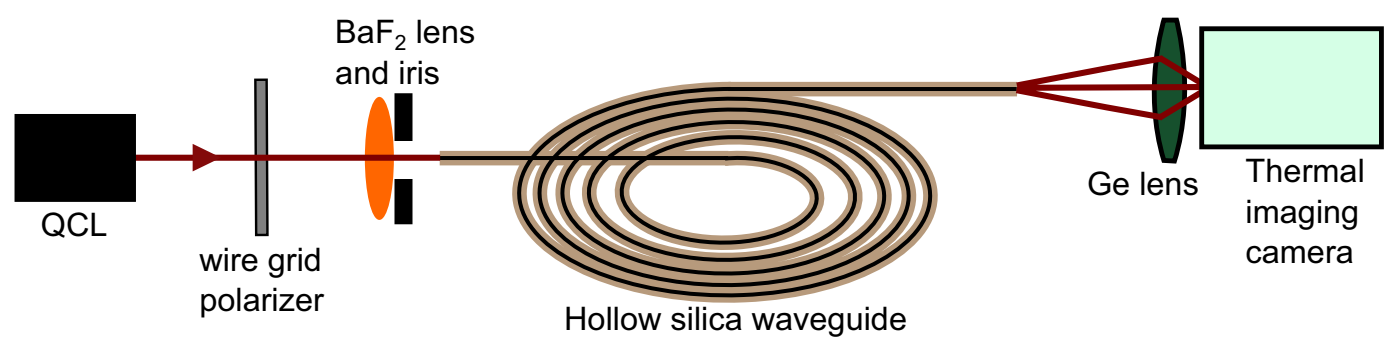

Fig. 7 Experimental arrangement for the investigation of the intensity distribution of light-exiting hollow silica waveguides 

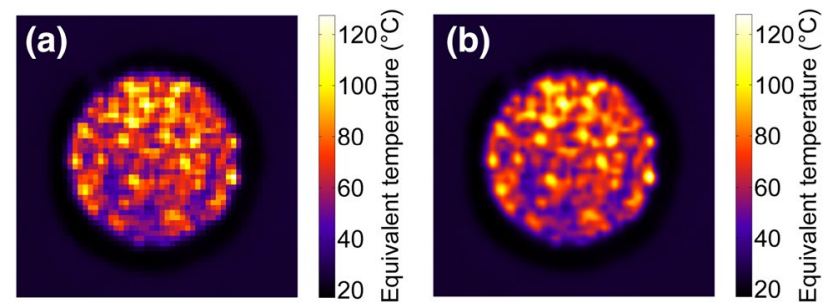

Fig. 8 a A typical thermal image recorded at the output of a coiled $1000 \mu \mathrm{m}$ bore waveguide transmitting the QCL beam and $\mathbf{b}$ the thermal image after up-sampling and interpolation to remove the pixellation

$40 \%$ responsivity at the $7.8 \mu \mathrm{m}$ of the QCL. Figure 7 shows the experimental configuration that was used.

Light exiting the QCL was coupled into the waveguide using a barium fluoride $\left(\mathrm{BaF}_{2}\right)$ lens that is mounted with an iris so that the $f$-number can be varied. The wire-grid polarizer was used to control the intensity to avoid saturating the camera. The germanium imaging lens has a focal length of $25 \mathrm{~mm}$ and was sufficient to image the output face of the larger waveguide with good spatial resolution.

\subsection{Interpretation of thermal images of QCL intensity distributions}

A typical image recorded with the thermal imaging camera located at the output of the larger waveguide is shown in Fig. 8a. The length of the waveguide is $5 \mathrm{~m}$, and it was coiled at a radius of approximately $15 \mathrm{~cm}$. The image in Fig. 8a is inherently pixellated due to the limited spatial resolution available when imaging the waveguide output onto a sensor array with a low pixel count. To remove the pixellation and enhance the aesthetic quality of the images, the data were up-sampled by a factor of ten and filtered using bi-cubic interpolation. Bi-cubic interpolation replaces a pixel value with a weighted average of a $4 \times 4$ neighbourhood. Figure 8 b shows the data of Fig. $8 \mathrm{a}$ after processing in this fashion. Radiation incident on the sensor array is digitized into camera count values by an analogue-to-digital converter with 14-bit resolution. A series of calibration curves are then used to convert the digital counts into total thermal radiance values, which are then converted to temperature $T_{\mathrm{obj}}$ using the total radiation law [30, 31].

$T_{\mathrm{obj}}=\frac{1}{\varepsilon \tau} W_{\mathrm{tot}}-\frac{1-\varepsilon}{\varepsilon} T_{\mathrm{amb}}-\frac{1-\tau}{\varepsilon \tau} T_{\mathrm{atm}}$

where $W_{\text {tot }}$ is the total thermal radiance, $T_{\mathrm{amb}}$ and $T_{\mathrm{atm}}$ are the ambient and atmospheric temperatures, and $\varepsilon$ and $\tau$ are the emissivity and atmospheric transmissions, respectively. The emissivity, atmospheric transmission and ambient
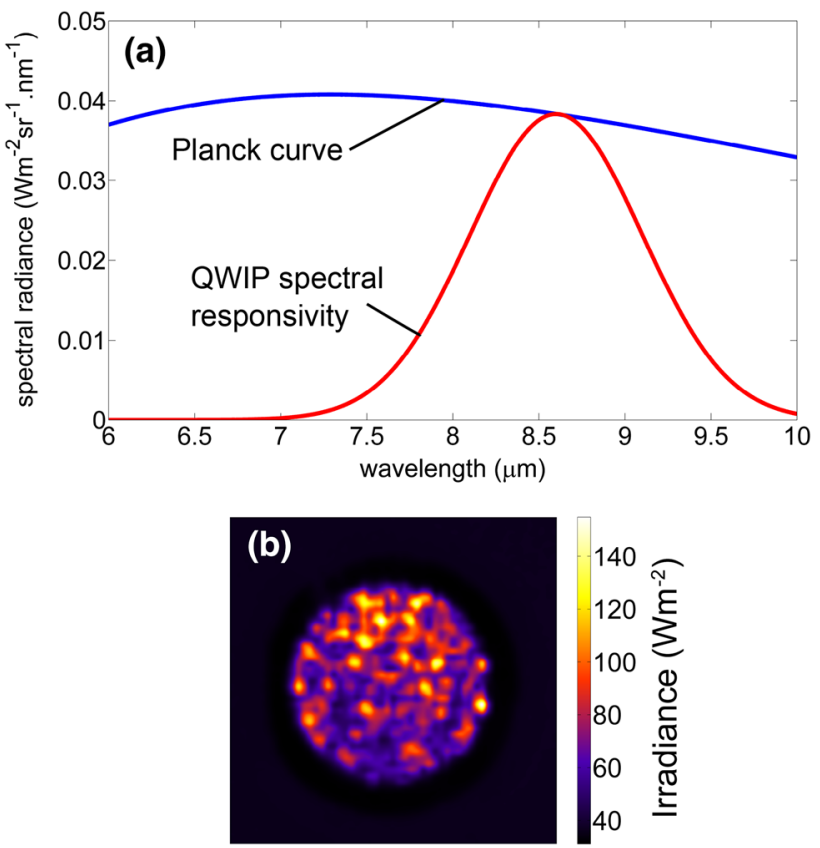

Fig. 9 a The spectral radiance distribution at $125^{\circ} \mathrm{C}$ and the Gaussian function representing the spectral responsivity of the thermal imaging camera and $\mathbf{b}$ the same data as in Fig. 8 displaying calculated irradiance values obtained from Planck curves calculated for each pixel's temperature value

and atmospheric temperature values that were used were $\varepsilon=0.98, \tau=0.9955$ and $T_{\text {amb }}=T_{\text {atm }}=295.1 \mathrm{~K}$, respectively. The values output by the camera are therefore equivalent temperature values, such as those in Fig. 8, and are related to the irradiance reaching the sensor. For this application, it is more relevant to know the irradiance at each pixel, rather than temperature. The total irradiance can be calculated by integrating the Planck distribution over all wavelengths, which results in the Stefan-Boltzmann law

$I=\sigma T^{4}$

where $\sigma=5.67 \times 10^{-8} \mathrm{Js}^{-1} \mathrm{~m}^{-2} \mathrm{~K}^{-4}$ is the Stefan-Boltzmann constant. The thermal imaging camera, however, is only sensitive to certain wavelengths which are defined by its spectral responsivity, which can be approximated by a Gaussian distribution with a full-width half-maximum (FWHM) of $1.4 \mu \mathrm{m}$ centred at $8.6 \mu \mathrm{m}$. Calculating the spectral radiance for a pixel and integrating the area under this Gaussian function yield the irradiance at that pixel. Figure 9a shows an example of the spectral radiance curve calculated for a temperature of $125^{\circ} \mathrm{C}$ (the peak temperature recorded in Fig. 8). Figure $9 \mathrm{~b}$ shows the same data as Fig. 8 with the irradiance calculated for each pixel using the method described above. The dependence of the irradiance on the temperature to the fourth power can be seen 
in the increased contrast of the image in Fig. $9 \mathrm{~b}$ relative to that in Fig. 8b.

The intensity distribution that is observed is a speckle pattern with high spatial frequency (in the context of this work) as indicated by the large number of small features. The speckle pattern is produced by the interference between the different modes populated in the waveguide. The dark outer ring that can be seen is the silica layer of the hollow waveguide.

The effect of ambient temperature on the mode pattern was tested by warming the waveguide with a heat gun. No observable change in the pattern was seen, suggesting that the waveguide has good thermal stability. Mechanical stability was investigated by manually perturbing the waveguide, and the pattern was observed to fluctuate with the induced vibration [see attached multimedia files]. This issue of mechanical instability does not pose too significant a problem for spectroscopy using the intra-pulse technique, however, due to measurements being made across a single pulse with a duration of the order of hundreds of nanoseconds, which is much shorter than the time frame of the mechanical perturbations.

\subsection{Influence of pulsewidth}

When driven using the intra-pulse technique, the laser emission experiences a frequency down-chirp across the pulse. It might therefore be expected that the mode pattern might change due the increase in the number of constituent wavelengths as the pulsewidth increases. Figure 10 shows a series of images recorded with different pulsewidths. The obvious difference between them is the increase in signal magnitude due to the integration of more energy over the exposure time of the camera. However, there is no observable difference in the mode pattern with increasing pulsewidth. This may be because the wavelength variation across the pulse ( $\sim 9 \mathrm{~nm}$ for a $500-\mathrm{ns}$ pulse) is too small to cause any significant visible change in the mode distribution.

\subsection{Influence of launch conditions and bore diameter}

The results of the analysis of Sect. 3 indicate that the number of modes should decrease with decreasing bore diameter and should vary with $f$-number of the coupling lens, with an optimum $f$-number for each bore diameter. In a thermal image, a reduction in the number of modes would be made apparent with a reduction in the spatial frequency of the mode distribution pattern. A series of $\mathrm{BaF}_{2}$ coupling lenses with focal lengths of 30,50 and $150 \mathrm{~mm}$ were used in the configuration shown in Fig. 7 in conjunction with an adjustable iris to obtain a range of launch $f$-numbers. A large number of modes are present

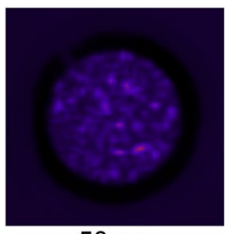

$50 \mathrm{~ns}$

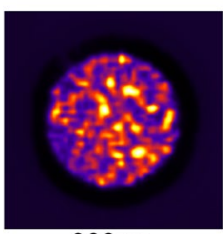

$300 \mathrm{~ns}$

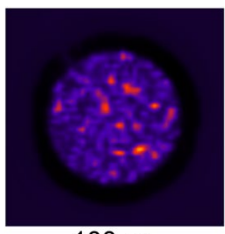

$100 \mathrm{~ns}$

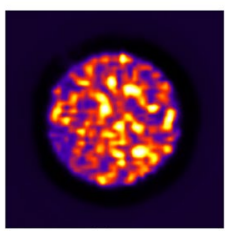

$400 \mathrm{~ns}$

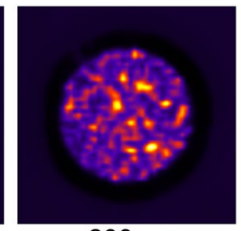

$200 \mathrm{~ns}$

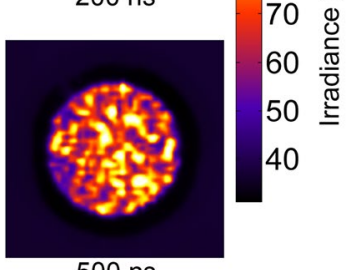

$500 \mathrm{~ns}$
Fig. 10 A series of images obtained for a range of QCL pulsewidths. No obvious change in the mode distribution pattern can be seen, only an increase in signal level with pulsewidth
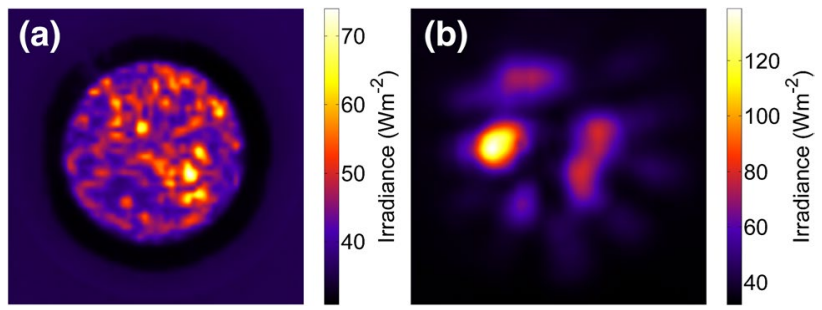

Fig. 11 Intensity distribution recorded a at the output of the $1000 \mu \mathrm{m}$ waveguide using $f / 30$ launch optics and $\mathbf{b}$ at the output of the $300 \mu \mathrm{m}$ waveguide using $f / 20$ launch optics. The reduction in the spatial frequency of the pattern in $\mathbf{b}$ relative to a suggests fewer supported modes

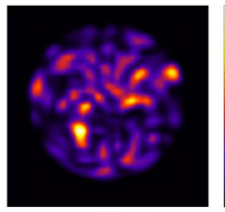

$f / 10$

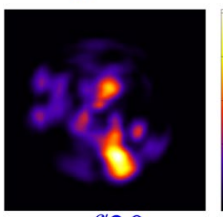

$f / 30$

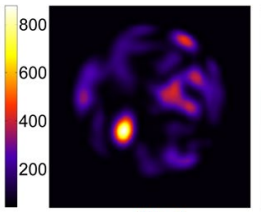

$f / 16$

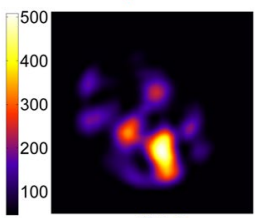

$f / 50$

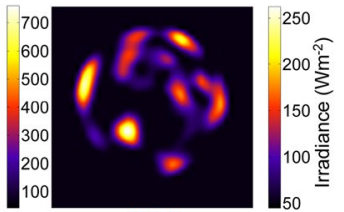

$f / 25$

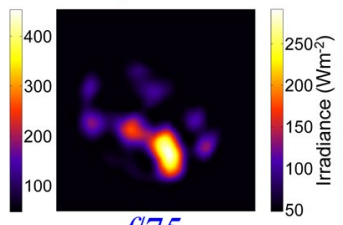

$f / 75$
Fig. 12 Intensity distribution patterns recorded for a range of launch $f$-numbers at the output of a $67-\mathrm{cm}$ straight length of the $1000 \mu \mathrm{m}$ waveguide. The images in the top row were obtained using a $50-\mathrm{m}$ focal length lens (red font), and the images in the bottom row were obtained using a $150-\mathrm{mm}$ lens (blue font)

when using the $1000 \mu \mathrm{m}$ bore waveguide, as can be seen by the fairly high spatial frequency in the patterns 
shown in Figs. 8, 9 and 10. Varying the $f$-number of the coupling lens altered the pattern, but no visible change in the spatial frequency of the pattern was observed. A reduction in the spatial frequency content is observed in the images obtained when using the $300 \mu \mathrm{m}$ waveguide relative to the $1000 \mu \mathrm{m}$ waveguide, as shown in Fig. 11. Figure 11a shows the output intensity distribution from the $1000 \mu \mathrm{m}$ waveguide using an $f / 30$ launch, and Fig. 11b from the $300 \mu \mathrm{m}$ waveguide using an $f / 20$ launch. Since the output face of the $300 \mu \mathrm{m}$ waveguide is so small, the image was defocused slightly to increase the size of the image and the number of illuminated pixels. Adjusting the $f$-number of the coupling lens again altered the pattern; however, no visible change in the spatial frequency was observed.

This was believed to be due to the coiled nature of the 5-m-long waveguide, which causes different modes to populate as the launch conditions are varied, thus a change in the pattern but no change in the overall number of modes. The experiments were therefore repeated using straight sections of waveguide. A ceramic cleaving tool was used to cut through the acrylate buffer and score an indentation into the silica wall. Application of an axial force splits the waveguide and results in a smooth end face. The lengths of the cleaved sections were $67 \mathrm{~cm}$ for the $1000 \mu \mathrm{m}$ waveguide and $98 \mathrm{~cm}$ for the $300 \mu \mathrm{m}$ waveguide. Figure 12 shows the results of varying launch $f$-number for the straight $1000 \mu \mathrm{m}$ waveguide. The top row of images was obtained using a 50-mm coupling lens and the bottom row was obtained using a $150-\mathrm{mm}$ coupling lens. The spatial frequency content within the images, and therefore, the number of supported modes is observed to decrease as the $f$-number of the launch optic increases, as predicted by Fig. 4b. The decrease in overall signal level per row as the $f$-number is increased is because of clipping of the beam by the iris as it is stopped down. The increase in irradiance of these images relative to the previous ones is due to the shorter length of the waveguide through which the light propagates, meaning that attenuation is substantially reduced. Also, now that the waveguide is aligned straight there are no bend losses.

In order to quantify the reduction in the number of supported modes, the spatial frequency was calculated for each of the images in Fig. 12. A reduction in spatial frequency is expected as $f$-number increases (up to around $f / 64$ based on the analysis of Sect. 3) as fewer modes contribute to the interference creating the speckle pattern. The spatial frequency of an image can be calculated using the Fourier transform, implemented using the two-dimensional fast Fourier transform (FFT) algorithm. Prior to transformation, the images were scaled to the same mean value and normalized to reduce bias towards regions of high irradiance. In image processing, normalization is also referred to as

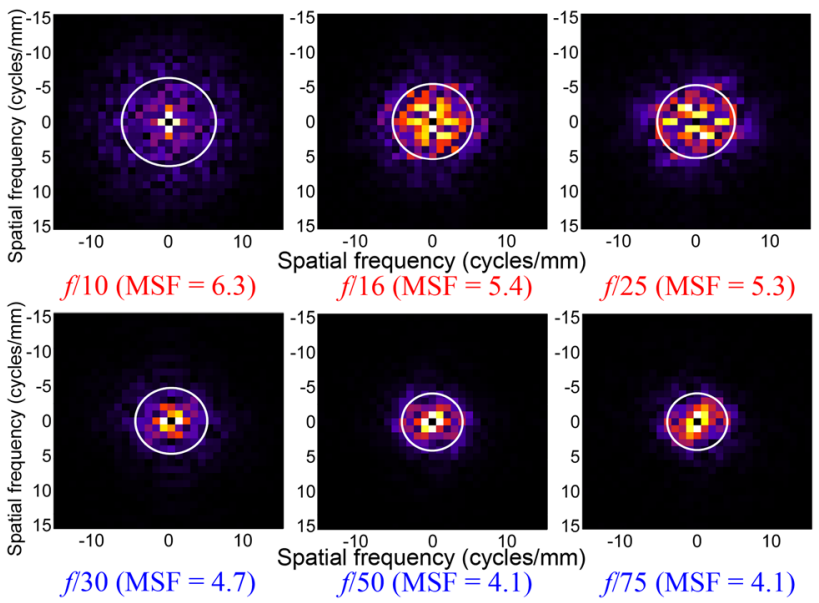

Fig. 13 Fourier transforms of intensity distributions recorded through $1000 \mu \mathrm{m}$ bore diameter HSW. The rings indicate the mean spatial frequency (MSF) in each image, which is given numerically in brackets below each

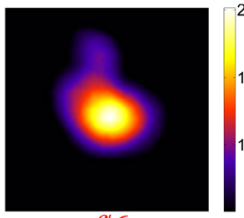

$f / 6$

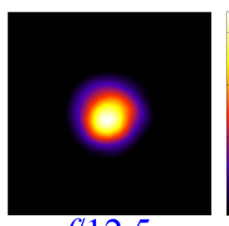

$f / 12.5$

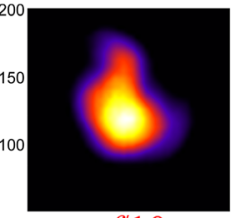

$f / 10$

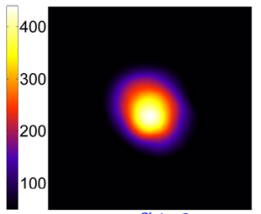

$f / 16$

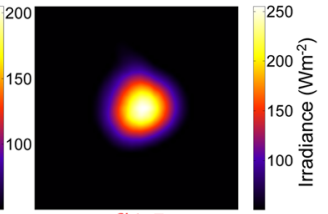

$f / 15$

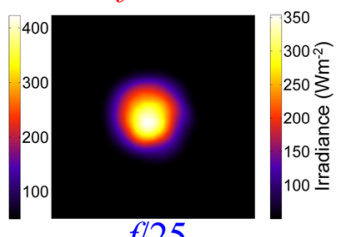

$f / 25$
Fig. 14 Intensity distribution patterns recorded for a range of launch $f$-numbers recorded at the output of a $98-\mathrm{cm}$ straight length of the $300 \mu \mathrm{m}$ waveguide. The images in the top row were obtained using the $30-\mathrm{mm}$ lens (red font), and the images in the bottom row were obtained using the $50 \mathrm{~mm}$ lens (blue font)

contrast or histogram stretching and is used to ensure that the full range of greyscale values are used, thereby enhancing contrast. The FFT was calculated, and a low-pass filter of 14 cycles/mm was applied to suppress the influence of high spatial frequencies, e.g. those associated with the pixels. The absolute magnitudes of the Fourier transforms of each of the images shown in Fig. 12 are shown in Fig. 13. The quadrants of the spectrum are repositioned so that the DC term at a spatial frequency of 0 cycles $/ \mathrm{mm}$ is at the centre. The DC pixel value corresponds to the sum of the irradiance values in the image and as a consequence is very high relative to other values. It is therefore set to zero here so that the other spatial frequencies, which are of interest, are visible in a scaled image. The rings in each image 


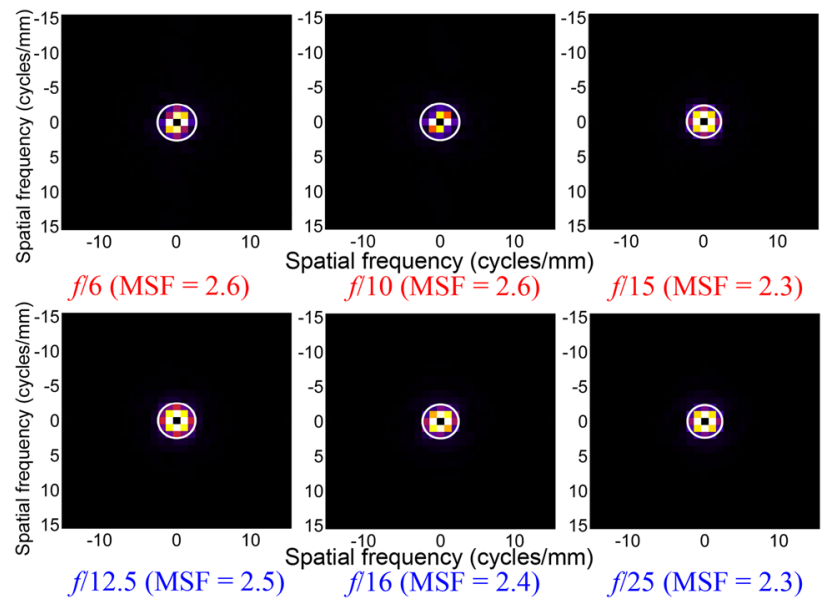

Fig. 15 Fourier transforms of intensity distributions recorded through $300 \mu \mathrm{m}$ bore diameter HSW. The rings indicate the mean spatial frequency (MSF) in each image, which is given numerically in brackets below each

correspond to the mean spatial frequency and can be seen to reduce with increasing $f$-number as predicted.

The $f$-number for optimum coupling to the lowest order $\mathrm{HE}_{11}$ mode was stated before as $f / 64$ for the $1000 \mu \mathrm{m}$ bore diameter waveguide, which can be set using the $150 \mathrm{~mm}$ lens. This indicates that the two images on the right of the bottom row (at $f / 50$ and $f / 75$ ) roughly correspond to the set-up required for the minimum number of modes achievable with this size waveguide. Figure 14 shows the results of varying launch $f$-number for the straight $300 \mu \mathrm{m}$ waveguide. The top row was obtained using a 30-mm coupling lens, and the bottom row was obtained using a $50-\mathrm{mm}$ coupling lens.

With this narrower bore waveguide, we can see the influence of higher-order modes at smaller $f$-numbers manifested by the distortion in the feature seen in the two images on the left side of the top row (at $f / 6$ and $f / 10$ ). At higher $f$-numbers, there is essentially single-mode transmission, with those images on the bottom row having particularly good modal purity. The overall signal level of the images in Fig. 14 is less than that of the images in Fig. 12 due to the $1 / a^{3}$ dependence of the attenuation on the bore diameter, as shown in Fig. 3. A pulsewidth of 200 ns was used for all the images in Figs. 12 and 14. The Fourier transforms of each of the intensity distributions shown in Fig. 14 were calculated in the same manner as described above, and the magnitudes of each are shown in Fig. 15. The mean spatial frequency for these distributions is significantly smaller than those of the $1000 \mu \mathrm{m}$ bore diameter waveguide, as expected since there is only a single feature in the image plane. The mean spatial frequency varies by only a small amount over the range of launch conditions, as expected since transmission is essentially single mode above approximately $f / 10$.
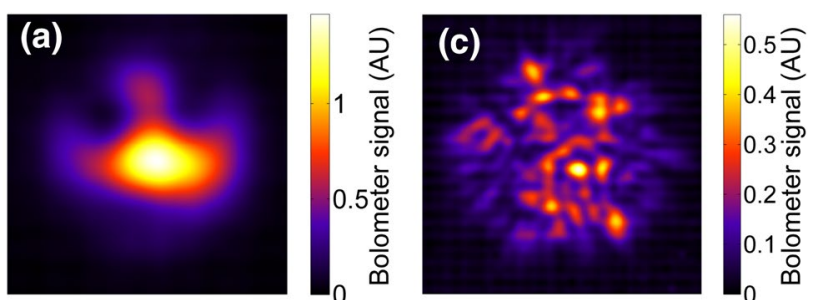

(b)

(d)
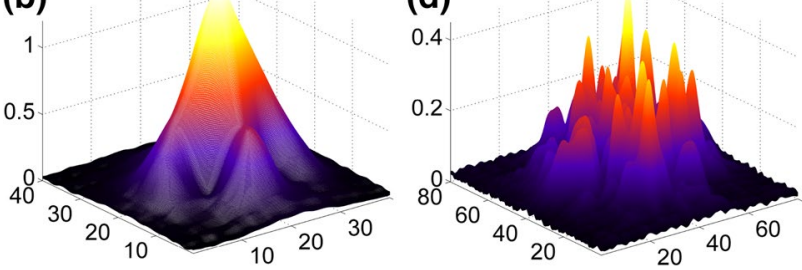

Fig. 16 Intensity distributions detected at the outputs of 5-m coiled lengths of the $300 \mu \mathrm{m}$ waveguide $\mathbf{a}$ and $\mathbf{b}$ and the $1000 \mu \mathrm{m}$ waveguide $\mathbf{c}$ and $\mathbf{d}$ using a micro-bolometer array beam profiler

\subsection{QCL intensity distributions measured with a beam profiler}

The outputs of the waveguides were also investigated using a micro-bolometer array beam profiler (DataRay Inc. WinCamD-FIR2-16-HR) as a comparison with the results obtained with the thermal imaging camera. The beam profiler sensor consists of $640 \times 480$ pixels with dimensions of $17 \mu \mathrm{m}^{2}$ and has a spectral response covering the region from $2 \mu \mathrm{m}$ to $16 \mu \mathrm{m}$. In this investigation, the beam profiler was placed at the output of the waveguide with the sensor located about $5 \mathrm{~mm}$ from the waveguide end face. No imaging optics were used, so light exiting the waveguide was allowed to diverge naturally to illuminate a region of the sensor array. This corresponded to about $40 \times 40$ pixels for the $300 \mu \mathrm{m}$ waveguide and approximately $80 \times 80$ pixels for the $1000 \mu \mathrm{m}$ waveguide. Intensity distributions obtained from the beam profiler are shown in Fig. 16 using 5-m coiled sections of the $300 \mu \mathrm{m}$ waveguide (a) and (b) and the $1000 \mu \mathrm{m}$ waveguide (c) and (d). One observation here is the apparent reduction in the number of modal features (reduced spatial frequency) in the bolometer results shown in Fig. 16 images relative to those obtained with the thermal imaging camera for 5-m coiled sections of waveguide shown in Figs. 8, 9 and 10. This suggests that more detailed resolution of the output intensity profile is achievable using the thermal imaging camera in combination with germanium imaging optics.

\section{Discussion and conclusions}

The transmission of quantum cascade laser light through hollow silica waveguides has been investigated. The 
influence of bore diameter and launch condition on the attenuation and modal properties of light within the waveguide was modelled using established equations with parameters specific to a $7.8-\mu \mathrm{m}$ QCL and commercially available HSWs. One result showed that modal attenuation is heavily dependent on the bore diameter, meaning that although smaller bore waveguides exhibit more loss than larger ones, they have a higher modal purity due to higher-order modes being more rapidly attenuated. This was verified in the thermal images where fewer modal features were observed in the output intensity distributions from $300 \mu \mathrm{m}$ bore waveguides relative to $1000 \mu \mathrm{m}$ bore waveguides. This is as expected, considering that attenuation occurs at each reflection at the internal coating interface. More reflections per unit length will occur for waveguides with a smaller bore diameter, and more reflections will occur for higher-order modes which couple at higher acceptance angles.

Another observation was that coupling of different modes is dependent on the $f$-number of the launch optic. Calculation of the overlap integral between the spatial profile of the Gaussian beam with the spatial profile of the first five hybrid modes for a range of launch conditions revealed the optimum launch $f$-numbers for maximum coupling to the lowest order mode and for lowest total loss, as shown in Fig. 4. The influence of launch $f$-number on the modal properties of the waveguide was investigated using a set of three coupling lenses in combination with an adjustable aperture, which allowed for a range of $f$-numbers from $f / 6$ to f/75. With 5-m-long coiled sections of waveguide, no change in the spatial frequency of the output distribution patterns was observed. With straight sections of waveguide, however, the modal content was observed to decrease with increasing $f$-number as predicted from the modelled results. Indeed, the classic single-mode Gaussian intensity distribution was observed at the output of the $300 \mu \mathrm{m}$ bore diameter waveguide with appropriate launch conditions. In fact, guided single-mode transmission should be achievable with longer wavelengths (or smaller bore diameters) of $10 \mu \mathrm{m}$ or greater, confirming the previously quoted rule-of-thumb of $\lambda>30 a$ [14], as has been observed previously [2].

With the present spectroscopy system with which the laser is used, the multimode nature of the HSW transmission is not a particular issue because the signal across all modes is integrated. Moreover, because the spectroscopic acquisition is so fast, measurements are made across laser pulses with durations of the order of hundreds of nanoseconds, no speckle noise associated with inter-mode interference is expected in the time domain. This can be verified by investigating the spectral background for the two waveguides in the same manner as [17]. Normalized absorptions obtained from laser pulses transmitted through the two waveguides and additionally through a conventional

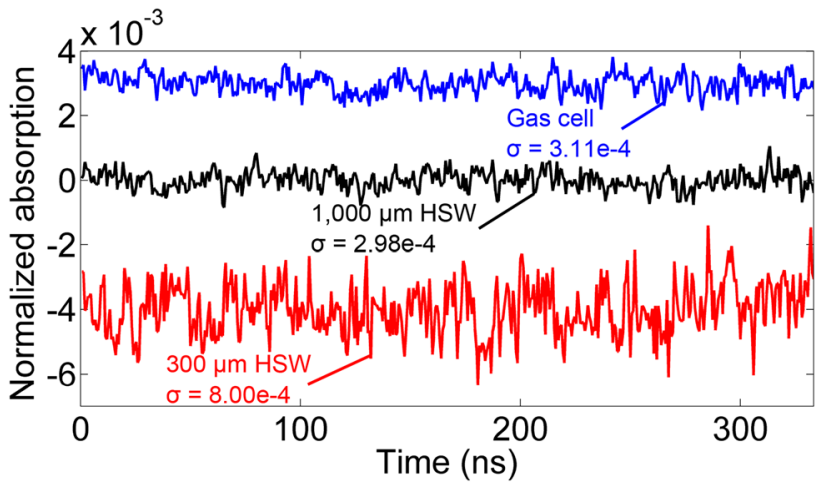

Fig. 17 Normalized absorptions calculated from QCL pulses transmitted through a conventional gas cell, the $1000 \mu \mathrm{m}$ HSW and the $300 \mu \mathrm{m}$ HSW. The standard deviation $(\sigma)$ of the data indicates the relative noise level. The gas cell data and $300 \mu \mathrm{m}$ HSW data are offset for clarity

gas cell are shown in Fig. 17. The noise present is primarily detector noise, and there is no significant change in noise level between the gas cell and the $1000 \mu \mathrm{m}$ bore diameter waveguide with standard deviations of $\sigma=3.11 \times 10^{-4}$ and $\sigma=2.98 \times 10^{-4}$, respectively. There is an increase in noise level in the $300 \mu \mathrm{m}$ bore diameter waveguide $\left(\sigma=8.00 \times 10^{-4}\right)$; however, this can be attributed to the diameter dependent attenuation.

Multimode transmission may, however, become a problem in cases where particularly long waveguides or highbandwidth detectors are used because dispersive degradation of the signal could occur. In these cases, it may be beneficial to select the source wavelength and bore diameter, and launch optics carefully in order to ensure guided single-mode transmission.

Acknowledgments The authors would like to acknowledge support from the Technology Strategy Board (project ref 100846) and the Engineering and Physical Sciences Research Council (EPSRC EP/ H02252X). Additionally, the authors would like to thank the EPSRC Engineering Instrument Pool for the loan of the FLIR thermal imaging camera and Peter Brunt of Laser2000, UK, for the use of the DataRay micro-bolometer beam profiler (For enquiries relating to the access to the research data or other materials referred to in this article, please contact Cranfield University Library and Information Services-library@cranfield.ac.uk)

Open Access This article is distributed under the terms of the Creative Commons Attribution License which permits any use, distribution, and reproduction in any medium, provided the original author(s) and the source are credited.

\section{References}

1. J.A. Harrington, Fiber Integr Opt. 19, 211-227 (2000)

2. Y. Matsuura, T. Abel, J. Hirsch, J.A. Harrington, Electron. Lett. 30(20), 1688-1690 (1994) 
3. R. George, J.A. Harrington, Appl. Opt. 44(30), 6449-6455 (2005)

4. C.M. Bledt, J.A. Harrington, J.M. Kriesel, Proc. SPIE 8218, 82180H-1-10 (2012)

5. J.A. Harrington, Infrared Fiber Optics and Their Applications (SPIE press, Bellingham, 2004)

6. Polymicro Technologies ${ }^{\mathrm{TM}}$, Phoenix AZ http://www.molex.com/ molex/products/group?channel=products \&key=polymicro. Accessed 06 June 2014

7. C.M. Charlton, B.T. Thompson, B. Mizaikoff, in Frontiers in Chemical Sensors: Novel Principles and Techniques, ed by O.S. Wolfbeis, G. Orellana, M.C. Moreno-Bondi (Springer, Berlin, 2005)

8. J. Faist, F.D.L. Capasso, S.C. Sirtori, A.L. Hutchinson, A.Y. Cho, Science 264, 553-556 (1994)

9. F. Capasso Opt. Eng. 49:11, 111102-1-9 (2010)

10. C. Charlton, F. de Melas, A. Inberg, N. Croitoru, B. Mizaikoff, IEE Proc. Optoelectron. 150, 4 (2003)

11. P.R. Fortes et al, Proceedings of the SPIE 8570, 85700Q-1-9 (2013)

12. R.K. Nubling, J.A. Harrington, Opt. Eng. 37(9), 2454-2458 (1998)

13. M. Miyagi, S. Kawakami, J. Lightwave Technol. LT-2 116-26 (1984)

14. C.M. Bledt, J.A. Harrington, J.M. Kriesel, Appl. Opt. 51(16), 3114-3119 (2012)

15. P. Patimisco, V. Spagnolo, M.S. Vitiello, A. Tredicucci, G. Scamarcio, C.M. Bledt, J.A. Harrington, Appl. Phys. B 108, 255$260(2012)$

16. A. Sampaolo, P. Patimisco, J.M. Kriesel, F.K. Tittel, G. Scamacio, V. Spagnolo, Opt. Exp. 23(1), 195-204 (2015)
17. J. Chen, A. Hengauer, R. Strzoda, M.C. Amman, Appl. Opt. 49(28), 5254-5261 (2010)

18. E. Normand, M. McCullogh, G. Duxbury, N. Langford, Opt. Lett. 28(1), 16-18 (2003)

19. J.P. Dakin, R.G.W. Brown, HandBook of Optoelectronics, vol. 1 (Taylor and Francis, Boca Raton, 2006)

20. E. Hecht, Optics, 4th edn. (Addison Wesley, San Francisco, 2002)

21. irflex.com IRF-SE chalcogenide longwave mid-infrared fiber. Accessed 7 Act 14

22. E.D. Palik, Handbook of Optical Constants of Solids (Academic press, Boston, 1985)

23. E.A.J. Marcatili, R.A. Schmeltzer, Bell Syst. Tech. J. 43, $1783-$ 1809 (1964)

24. R.E. Wagner, W.J. Tomlinson, Appl. Opt. 21(15), 2671-2688 (1982)

25. A. Hongo, M. Miyagi, K. Sakamoto, S. Karasawa, S. Nishida, Opt. Laser Technol. 19(4), 214-216 (1987)

26. R.M. Jenkins, R.W.J. Devereux, Appl. Opt. 31(24), 5086-5091 (1992)

27. Cascade Technologies ${ }^{\mathrm{TM}}$, Stirling, UK. http://www.cascade-technologies.com. Accessed 06/06/2014)

28. J. Hodgkinson, R.P. Tatam, Meas. Sci. Technol. 24, 1-59 (2013)

29. J. Manne, W. Jäger, J. Tulip, Appl. Phys. B. 94, 337-344 (2009)

30. Thermacam TM Researcher 2.7 user manual, FLIR systems (2003)

31. D. Bursell: Evaluation Engineering (2007) http://www.evaluationengineering.com/articles/200712/getting-the-most-fromyour-ir-camera.php. Accessed 25 July 14 